\title{
The bulge-disk orthogonal decoupling in galaxies: NGC 4698 and NGC 4672
}

\author{
By F. B ERT O L A ${ }^{1}$, E. M. C O R S I N I ${ }^{1}$, \\ M. CA P P ELLARI I. C. VEGA BELTRÁ N ${ }^{2}$, \\ A. PIZZELLA ${ }^{3}$, M. SARZI I AND J. G. FUNES S. J. ${ }^{1}$ \\ ${ }^{1}$ Dipartimento di Astronomia, Università di Padova, Vicolo dell'Osservatorio 5, I-35122 \\ Padova, Italy \\ ${ }^{2}$ Telescopio Nazionale Galileo, Osservatorio Astronomico di Padova, Vicolo dell'Osservatorio 5, \\ I-35122 Padova, Italy \\ ${ }^{3}$ European Southern Observatory, Alonso de Cordova 3107, Casilla 19001, Santiago 10, Chile
}

\begin{abstract}
We report the case of the geometrical and kinematical decoupling between the bulge and the disk of the Sa galaxy NGC 4698. The $R$-band isophotal map of this spiral shows that the bulge structure is elongated perpendicularly to the major axis of the disk. At the same time a central stellar velocity gradient is found along the major axis of the bulge. We also present the Sa NGC 4672 as good candidate of a spiral hosting a bulge and a disk orthogonally decoupled with respect to one other. This decoupling of the two fundamental components of a visible galaxy suggests that the disk could represent a second event in the history of early-type spirals.
\end{abstract}

\section{Introduction}

NGC 4698 is classified Sa by Sandage \& Tammann (1981) and Sab(s) by de Vaucouleurs et al. (1991, RC3). Sandage \& Bedke (1994, CAG) present NGC 4698 as an example of the early-to-intermediate Sa type since it is characterized by a large central bulge and tightly wound spiral arms. In addition to a remarkable geometrical decoupling between the bulge and the disk whose apparent major axes appear oriented in an orthogonal way at simple visual inspection of galaxy plates (see Panels 78, 79 and 87 in CAG), a spectrum taken along the minor axis of the disk shows the presence of a stellar velocity gradient which could be ascribed to the bulge. For this reason NGC 4698 is a noteworthy case for the study of the formation processes of disks in spirals.

\section{Results}

The $R$-band isophotal map of NGC 4698 (Fig. 1 left panel) shows the geometrical decoupling $\left(\Delta\right.$ P.A. $\left.\simeq 90^{\circ}\right)$ between the bulge and the disk of these spiral. The phenomenon is visible both in the inner isophotes and in the outermost one, which is characterized by two 'bumps' protruding perpendicularly to the galaxy major axis. The isophotes between $4^{\prime \prime}$ and $19^{\prime \prime}$ appear round in the plot. However as soon as an exponential disk is subtracted, they become elongated perpendicularly to the disk major axis. In order to disentangle between the light contribution of bulge and disk, we decomposed the surface-brightness radial profiles extracted along different axes of NGC 4698 as the sum of an $r^{1 / 4}$ bulge $\left(\mu_{e}=19.6 \mathrm{mag} \cdot \operatorname{arcsec}^{-2} ; r_{e}=11.3^{\prime \prime} ; q=1.1\right)$ and an exponential disk $\left(\mu_{0}=19.2 \mathrm{mag} \cdot \operatorname{arcsec}^{-2} ; r_{d}=32.2^{\prime \prime} ; i=60^{\circ}\right)$. The bulge results the dominating component inside $10^{\prime \prime}$ along the galaxy major axis and inside $14^{\prime \prime}$ along the minor axis. The axial ratio of the bulge is found to be greater than unity confirming its exceptional property to be elongated along the disk minor axis. Alternative non-parametric decompositions of the NGC 4698 surface-brightness distribution (Moriondo et al. 1998) 

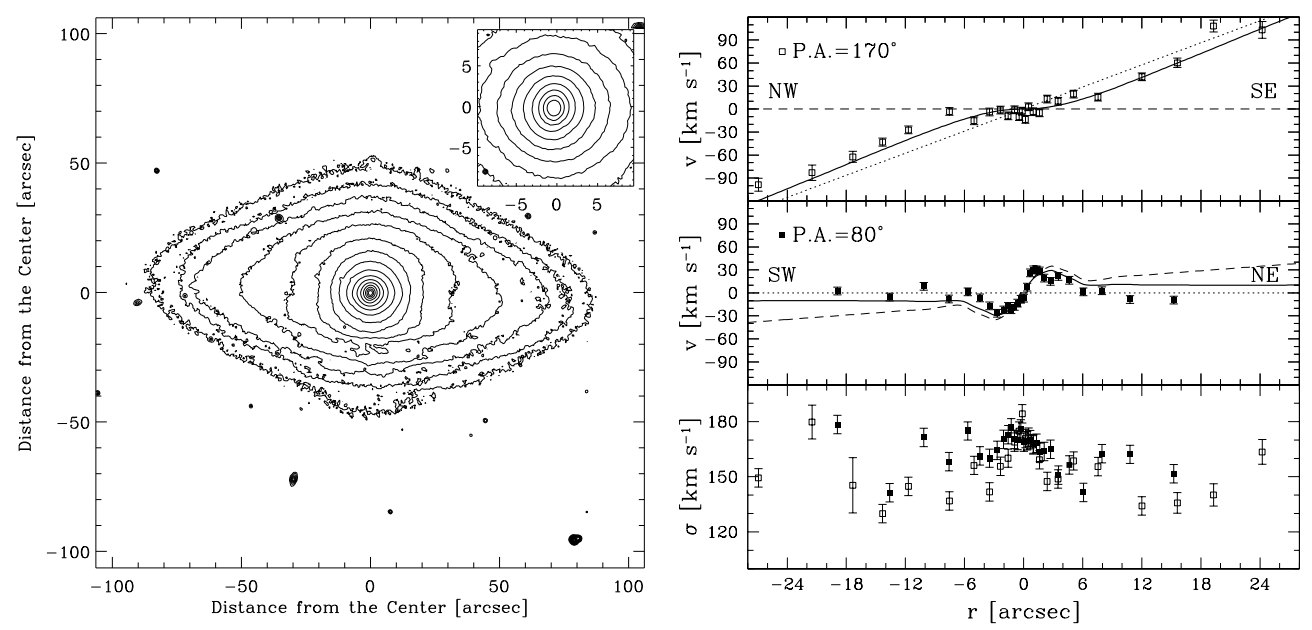

Figure 1. Left panel: $R$-band isophotes of NGC 4698 given in steps of $0.4 \mathrm{mag} \cdot \operatorname{arcsec}^{-2}$ with the outermost one corresponding to $21.8 \mathrm{mag} \cdot \operatorname{arcsec}^{-2}$ and the central one to $15.8 \mathrm{mag} \cdot \operatorname{arcsec}^{-2}$. In the inset the isophotal map of the inner $10^{\prime \prime}$ is plotted. North is to right and east up. Right panel: Observed stellar rotation velocity $\left(V_{\odot}=992 \pm 10 \mathrm{~km} \mathrm{~s}^{-1}\right)$ and velocity dispersion as a function of radius along the major (open squares) and minor (filled squares) axes of NGC 4698. The dashed and the dotted lines represent the velocity contribution of the bulge and disk components to the total velocity (thick continuous line) of our model

with a disk profile flattening toward the center would produce lower residuals when the modeled surface brightness is subtracted from the observed one. However our standard assumption of an $r^{1 / 4}$ bulge and an exponential disk does not affect the photometric and kinematical orthogonal decoupling between bulge and disk of NGC 4698 (Fig. 1).

The major-axis stellar velocity curve is characterized by a central plateau, indeed the stars have a zero rotation for $|r| \leq 8^{\prime \prime}$. At larger radii the observed stellar rotation increases from zero to an approximately constant value of about $200 \mathrm{~km} \mathrm{~s}^{-1}$ for $|r| \geq 50^{\prime \prime}$ up to the farthest observed radius at about $80^{\prime \prime}$. These velocities are in agreement within the errors with those measured by Corsini et al. (1999). The stellar velocity dispersion profile has been measured out to $30^{\prime \prime}$. It is peaked in the center at the value of 185 $\mathrm{km} \mathrm{s}^{-1}$. We measured the minor-axis stellar kinematics out to about $20^{\prime \prime}$ on both sides of the galaxy. In the nucleus the stellar velocity rotation increases to about $30 \mathrm{~km} \mathrm{~s}^{-1}$ at $|r| \simeq 2^{\prime \prime}$, decreasing to zero further out. The velocity dispersion profile has a central maximum of $175 \mathrm{~km} \mathrm{~s}^{-1}$ in agreement with the value measured on the spectrum along the major axis. The stellar velocity curves and velocity-dispersion radial profiles (out only to $28^{\prime \prime}$ for the spectrum along the major axis) are plotted in Fig. 1 (right panel). In order to demonstrate that the observed velocity curves along the major and minor axis are consistent with the rotation of a bulge and a disk with perpendicular angular momenta we modeled the observed line-of-sight velocity distribution in the following way. Along the major axis we assumed a velocity curve for the bulge constant at zero velocity with a constant velocity dispersion at $\sigma_{b}=160 \mathrm{~km} \mathrm{~s}^{-1}$, while for the velocity curve of the disk we assumed the velocity rising linearly to match the outer points of the plotted curve where the light contribution of the bulge is negligible, with a constant velocity dispersion at $\sigma_{d}=130 \mathrm{~km} \mathrm{~s}^{-1}$. The resulting velocity curve is obtained by fitting with a Gaussian the sum of the two velocity components corresponding to the bulge and the disk weighted according to the photometric decomposition. The agreement with the observed points 
is very good and the flat central part of the observed velocity curve is well reproduced. A similar approach has been applied to reproduce the velocity curve along the minor axis. A constant zero velocity and a constant velocity dispersion $\sigma_{d}=130 \mathrm{~km} \mathrm{~s}^{-1}$ have been assumed for the disk, as well as a constant velocity dispersion $\sigma_{b}=160 \mathrm{~km} \mathrm{~s}^{-1}$ for the bulge. The bulge rotation has been maximized in such a way that the resulting velocity curve is after folding within the scatter of the data. It reaches a maximum of $35 \mathrm{~km} \mathrm{~s}^{-1}$ in the inner $3^{\prime \prime}$ decreasing to a local minimum of $15 \mathrm{~km} \mathrm{~s}^{-1}$ at $|r| \simeq 6^{\prime \prime}$ and then increasing afterwards.

\section{Discussion and conclusion}

The observed stellar kinematics can be interpreted as due to an orthogonal kinematical decoupling between the bulge and disk components. Assuming that the intrinsic shape of a bulge is generally triaxial (Bertola, Vietri \& Zeilinger 1991), and that the plane of the disk coincides with the plane of the bulge perpendicular either to the major or to the minor axis, we deduce that the observed configuration indicates that the major axis of the bulge is perpendicular to the disk, given that the disk is seen not far from edge on. The fact that the velocity field of the bulge is characterized by a zero velocity along its apparent minor axis, as indicated by the central plateau in the rotation curve along the disk major axis, and by a gradient along its major axis suggests that the rotation axis of the bulge lies on the plane of the disk.

Our photometric and spectroscopic data and the ensuing interpretation of the orthogonal decoupling between the bulge and disk of NGC 4698 can be explained if the disk has formed in a distinct process occurred in the history of the galaxy. We suggest that the disk has formed at a later stage due to acquisition of material by a pre-existing triaxial spheroid on its principal plane perpendicular to the major axis. An example of acquisition on the plane perpendicular to the minor axis could be represented by NGC 7331 (Prada et al. 1996), where the bulge has been found counterrotating with respect to the disk. Up to now NGC 4698 and NGC 7331 represent the only cases of kinematical evidence that disk galaxies with prominent bulge could be started as 'undressed spheroid' and their disks accreted gradually over several billion years, as suggested by Binney \& May (1986). Recently such kind of processes have been considered within semi-analytical modeling techniques for galaxy formation, where the disks accrete around bare spheroids previously formed (e.g. Kauffmann 1996; Baugh, Cole \& Frenk 1996). In this framework polar-ring elliptical galaxies like NGC 5266 (Varnas et al. 1987) and ellipticals with dust lanes along the minor axis (Bertola 1987) could be a transient stage towards the formation of spiral systems like NGC 4698.

In order to verify if the acquisition phenomena giving rise to NGC 4698 and NGC 7331 are general processes of galaxy formation we need to know how these objects are unique or peculiar. To address this question we began a photometric and spectroscopic survey of early-type spirals even with a slight indication of geometrical orthogonal decoupling between bulge and disk. The finding of galaxies with round bulges suggests that the case of the orthogonal geometrical decoupling between the bulge and disk of NGC 4698 could be a more general phenomenon. In fact, even a bulge which appears round on a plate becomes intrinsically elongated after the subtraction of an inclined exponential disk. The further spectroscopic analysis of this kind of objects with the slit of the spectrograph set along the major axis of their bulges allow to determine if the geometrical decoupling is associated with the kinematical decoupling, as in NGC 4698. As first result of this survey we present the case of NGC 4672 (Sarzi et al. 1999, in preparation). It is very probably a highly-inclined Sa spiral with a prominent $r^{1 / 4}$ bulge sticking out from the 

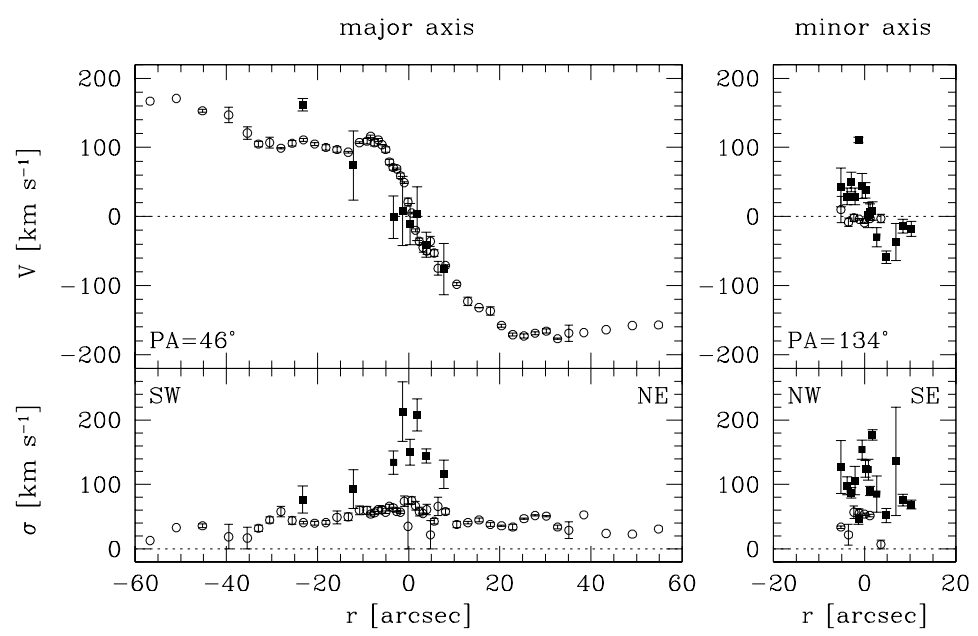

FiguRE 2. The stellar (filled squares) and ionized gas (open circles) kinematics measured along the major $\left(\right.$ P.A. $\left.=46^{\circ}\right)$ and minor axis $\left(\right.$ P.A. $=134^{\circ}$ ) of NGC 4672 . The systemic velocity is $V_{\odot}=3275 \pm 20 \mathrm{~km} \mathrm{~s}^{-1}$

plane of the disk (e.g. Fig. 3i by Whitmore et al. 1990) rather than a polar-ring galaxy. The stellar velocity field (Fig. 3) is characterized by the signature of a central zerovelocity plateau in the rotation curve obtained along the major-axis $\left(\right.$ P.A. $\left.=46^{\circ}\right)$ and by a steep velocity gradient observed along the minor axis $\left(\right.$ P.A. $\left.=134^{\circ}\right)$ similar to those measured in NGC 4698. In addition the major-axis gas rotation curve is typical of a disk in differential rotation and not of a ring. The close structural and kinematical resemblance of NGC 4672 to NGC 4698 makes the former a good candidate to be a new case of early-type spiral characterized by a bulge-to-disk orthogonal decoupling.

\section{REFERENCES}

Baugh, C.M., Cole, S. \& Frenk, C.S. 1996 MNRAS 283, 1361

Bertola, F. 1987, In Structure and Dynamics of Elliptical Galaxies T. de Zeeuw (ed.), Reidel, Dordrecht, p. 135

Bertola, F., Vietri, M. \& Zeilinger, W.W. 1991 ApJ 374, L13

Bertola, F., Cinzano, P., Corsini, E.M., et Al. 1996 ApJ 458, L67

Binney, J.J. \& MAY, A. 1986 MNRAS, 218, 743

Corsini, E.M., Pizzella, A., SArzi, M. ET Al. 1999, A\&A, in press astro-ph/9809366

De Vaucouleurs, G. ET AL. 1991 Third Reference Catalogue of Bright Galaxies. SpringerVerlag, New York (RC3)

Kauffmann, G. 1996 MNRAS 281, 487

Moriondo, G., Giovanardi, C. \& Hunt, L.K. 1998 A $E A S$ 130, 81

Prada, F., Gutierrez, C.M., Peletier, R.F. \& McKeith, C.D. 1996 ApJ 463, L9

SAndage, A. \& Bedke, J. 1994 The Carnagie Atlas of Galaxies. Carnagie Institution, Flintridge Foundation, Washington (CAG)

Sandage, A. \& Tammann, G.A. 1981 A Revised Shapley-Ames Catalog of Bright Galaxies. Carnagie Institution, Washington (RSA)

Varnas, S.R., Bertola, F., Galletta, G. et Al. 1987 ApJ 313, 69

Whitmore, B.C., Lucas, R.A., McElroy, D.B., et Al. 1990 AJ 100, 1489 